\title{
Pre-operative assessment for sleep apnoea in patients referred for bariatric surgery : the usefulness of various clinical screening tools
}

\author{
C Martinou1, UA Nayak1, GI Varughese', A Thomas'2, M Allen², L Varadhan' \\ 1Dept of Diabetes \& Endocrinology, ${ }^{2}$ Dept of Respiratory Medicine \\ University Hospitals of North Midlands NHS Trust, Stoke on Trent, UK
}

\section{Introduction}

- Obstructive sleep apnoea (OSA) is widely prevalent with obesity; its effect on OSA is multifactorial

- 1 SD increase in BMI increases risk of OSA by 4 fold (Young et al, NEJM 1993)

- The prevalence of OSA correlates well with the BMI; however the severity of OSA does not correlate well to the BMI in severely obese; the risk of severe OSA in these patient is as high as $95 \%$ (Serafini et al, Obes surgery 2001)

- There is a significant overlay of OSA with Obesity hypoventilation syndrome (OHS) in this cohort and it is common to assume that managing one could complement the other

- Epworth sleepiness scale (ESS) is a common screening tool for day time sleepiness, a complication of OSA

- Good at predicting OSA but not predicting its severity (Serafini, Obes Surg 2001)

- Good positive correlation with AHI index (Yeh, Obes Surg 2010) -STOP BANG

- in general practice a cut off of 3 is used; in bariatric practices generally a cut off of 5 is recommended

- $\geq 5$ correlated well with severe to very severe OSA (Chung, BJA 2011)

- Due to the evolving and dynamic nature of the bariatric service and modifications to its framework at our LHE, the guidelines at our hospital included a wide range of different clinical practice with time. This includes a combination of

- $A B G$ : with triggers of saturation $<92 \%$ or $\mathrm{pO} 2<8$ or $\mathrm{pCO} 2>6$

- STOP BANG Score

- Epworth Sleepiness score (ESS) $>10$

- Baseline spirometry

\section{Aim}

The aim of our retrospective analysis was to assess the utility of various clinical parameters used as screening test for OSA, by estimating their sensitivity against polysomnography-confirmed cases of OSA, in patients referred for bariatric surgery

\section{Methods}

- All patients seen in medical bariatric clinic over 12 months included

- Patients on established CPAP were excluded

- Various screening tests based on locally agreed guidelines were carried out on a group of these patients based on clinician's decision including bariatric physicians and surgeons and nurse specialist

- Retrospective observational analysis of standard clinical practice

- Patients with abnormal results were referred for formal sleep studies

- ABG ( based on pCO2>6, pO2, Sats on air $<92 \%$ )

- STOP BANG score>3

- $E S S>10$

- FEV1/NC $<70 \%$

\section{Results}

- A total of 227 patients had been seen in the endocrine clinics in 12 months - Mean BMl 47.6kg/m² (28-82)

- 39 patients had OSA already diagnosed and on OSA therapy

- $\mathrm{N}=104$ were assessed for OSA based on clinical index of suspicion

-35/104 (34\%) did not have OSA on formal sleep studies

\section{Results}

- $54 \%$ had severe OSA requiring CPAP treatment

- $12 \%$ had mild OSA which was conservatively managed

- $34 \%$ did not have any sleep apnoea

- There was a small but positive correlation between BMI and presence of OSA $(r=0.2)$ and the severity of OSA $(r=+0.15)$

\begin{tabular}{|l|c|c|c|c|c|}
\hline Tests & $\mathbf{N}=$ & $\begin{array}{c}\text { Sensitivity } \\
\%\end{array}$ & $\begin{array}{c}\text { Specificity } \\
\%\end{array}$ & $\begin{array}{c}\text { PPV } \\
\%\end{array}$ & $\begin{array}{c}\text { NPV } \\
\%\end{array}$ \\
\hline Sats $<92 \%$ & 72 & 8 & 86 & 57 & 30 \\
\hline pC02 $>6$ & 77 & 20 & 87 & 79 & 30 \\
\hline p02 $<8.0$ & 77 & 11 & 86 & 67 & 28 \\
\hline pO2<10 & 77 & 71 & 55 & 80 & 43 \\
\hline STOPBANG $\geq 3$ & 39 & 100 & 7 & 66 & 100 \\
\hline STOPBANG $\geq 5$ & 39 & 76 & 50 & 73 & 54 \\
\hline ESS $>10$ & 74 & 33 & 59 & 31 & 21 \\
\hline FEV1/VC $<70$ & 43 & 7 & 77 & 40 & 26 \\
\hline
\end{tabular}

\section{Discussion}

- The various commonly used history and clinical screening tests have limited sensitivity in diagnosing OSA

- There is always a discussion regarding the need to investigate OSA as this is likely to improve after bariatric surgery

- OSA is multifactorial and mere weight loss after bariatric surgery may not resolve this (Ashrafian et al, Thorax 2011)

- Establishment of a diagnosis of OSA is important as this has significant anaesthetic implications during peri-operative management

- Patients with OSA are more sensitive to opioid analgesics and hence risk of respiratory failure

- Re-establishment on CPAP is vital after bariatric surgery to safely wean the patient off the ventilator during surgery

- OSA takes longer to resolve after bariatric surgery - it is therefore vital that post-operative follow up is also arranged for these patients (Sjostrom et al, NEJM 2007)

- OSA related disordered breathing and related hypoxemia may have impacts on wound healing and long term cardiovascular health

\section{- Limitations of our analysis}

- Not all patients referred to the clinic were assessed - some patients were deemed not to have OSA clinically

- Many of the guidelines reported were based on local surgical and anaesthetics and hence not validated

\section{Conclusion}

- A significant proportion of patients have OSA

- Universal screening of all patients referred to bariatric services should be recommended, due to its wide prevalence and associated cardiovascular and respiratory morbidity

- It is important to appreciate that the various routinely used clinical screening tools only assess components of OSA and has limitations, thereby affecting their sensitivity and specificity

- Formal sleep oximetry studies are required, due to significant implications on anaesthetic and peri-operative management 